\title{
ON THE HILALI CONJECTURE FOR ODD GRADED HOMOTOPY GROUPS
}

\section{HASSAN AAYA, MOHAMED ANAS HILALI, MOHAMED RACHID HILALI and TARIK JAWAD}

\author{
University Hassan II \\ Casablanca \\ Morocco \\ e-mail: hassan.aaya@uic.ac.ma \\ rhilali@hotmail.fr \\ moahilali@gmail.com \\ j_tar@hotmail.fr
}

\begin{abstract}
The Hilali Conjecture (also known as conjecture H) [5] predicts that for any rationally elliptic and simply connected topological space $X$ we always have $\operatorname{dim}\left(\pi_{*}(X) \otimes \mathbb{Q}\right) \leqslant \operatorname{dim} H^{*}(X ; \mathbb{Q})$.

The goal of the present paper is to prove the Hilali conjecture for the topological spaces with odd homotopy groups satisfying some conditions, over the last years many works have tried to solve this conjecture in particular cases, e.g., [1], [4], [7], [8].
\end{abstract}

2010 Mathematics Subject Classification: 55P62.

Keywords and phrases: Hilali conjecture, rational homotopy, Sullivan minimal model, homotopy, cohomology, commutative differential graded algebra, elliptic space.

Received August 21, 2019; Revised October 17, 2019

(C) 2019 Scientific Advances Publishers 


\section{Introduction}

Let $X$ be a simply connected topological space, $X$ is called rationally elliptic if $\operatorname{dim}\left(\pi_{*}(X) \otimes \mathbb{Q}\right)<\infty$ and $\operatorname{dim}\left(H^{*}(X ; \mathbb{Q})\right)<\infty$.

Conjecture $\mathbf{H}$ (Topological version). Let $X$ be a rationally elliptic and simply connected topological space, then $\operatorname{dim}\left(\pi_{*}(X) \otimes \mathbb{Q}\right) \leqslant \operatorname{dim}\left(H^{*}(X\right.$; $\mathbb{Q}))$.

By the theory of minimal models of Sullivan [2], the rational homotopy type of $X$ is encoded in a differential algebra $(A, d)$ called the minimal model of $X$. This is a free graded algebra $A=\Lambda V$, generated by a graded vector space $V=\bigoplus_{k \geqslant 2} V^{k}$, and with decomposable differential, i.e., $d: V^{k} \rightarrow\left(\Lambda^{\geqslant 2} V\right)^{k+1}$, it satisfies:

$$
\left\{\begin{array}{l}
V^{k}=\left(\pi_{k}(X) \otimes \mathbb{Q}\right), \\
H^{k}(\Lambda V, d)=H^{k}(X, \mathbb{Q}) .
\end{array}\right.
$$

A well detailed chapter about Sullivan minimal models is contained in ([2], pp. 138-160). $X$ is rationally elliptic if and only if $(\Lambda V, d)$ is rationally elliptic.

Because of the correspondence between simply connected topological spaces and their minimal models the Hilali conjecture has an algebraic version:

Conjecture $\mathbf{H}$ (Algebraic version). If $(\Lambda V, d)$ is a Sullivan minimal model of a rationally elliptic and simply connected topological space $X$, then $\operatorname{dim} H^{*}(\Lambda V, d) \geqslant \operatorname{dim} V$.

The Hilali conjecture was formulated in 1990 [5] by Hilali who proved the case of rationally elliptic spaces of pure type, many other works ([1], [4], [5], [6], [7], [8]) proved the conjecture for several kind of topological 
spaces: H-spaces, nilmanifolds, symplectic and cosymplectic manifolds, coformal spaces with only odd-degree generators, 2 -stage spaces, formal and coformal spaces and for hyperelliptic spaces.

In the present paper, we start in Section 2 by recalling the Sullivan minimal models which are used in this work, in Section 3 we establish the main result: $\operatorname{dim} H^{*}(\Lambda V, d) \geqslant n$, where $V=\mathbb{Q}\left(a_{1}, \cdots, a_{n}\right)$ and $\left|a_{i}\right|$ are odd numbers satisfying $\left(d\left(a_{i}\right)\right)^{2}=d Q_{i}$ with $Q_{i} \in \Lambda\left(a_{1}, \cdots, a_{i-1}\right)$, our proof is based on a recurrence on $\operatorname{dim} V$. And finally in Section 4 we give an example to illustrating our theorem.

\section{Sullivan Minimal Models}

We recall some definitions and results about minimal models [2]. Let $(A, d)$ be a differential algebra, that is, $A$ is a (positively) graded commutative algebra over the rational numbers, with a differential $d$ which is a derivation, i.e., $d(a \cdot b)=(d a) \cdot b+(-1)^{\operatorname{deg}(a)} a \cdot(d b)$, where $\operatorname{deg}(a)$ is the degree of $a$. We say that $A$ is connected if $A^{0}=\mathbb{Q}$, and simply-connected if moreover $A^{1}=0$.

A simply-connected differential algebra $(A, d)$ is said to be minimal if:

(1) $A$ is free as an algebra, that is, $A$ is the free algebra $\Lambda V$ over a graded vector space $V=\oplus_{k \geq 2} V^{k}$, and

(2) For $x \in V^{k}, d x \in(\Lambda V)^{k+1}$ has no linear term, i.e., it lives in $\Lambda V^{>0} \cdot \Lambda V^{>0} \subset \Lambda V$.

Let $(A, d)$ be a simply-connected differential algebra. A minimal model for $(A, d)$ is a minimal algebra $(\Lambda V, d)$ together with a quasiisomorphism $\rho:(\Lambda V, d) \rightarrow(A, d)$ (that is, a map of differential algebras such that $\rho_{*}: H^{*}(\Lambda V, d) \rightarrow H^{*}(A, d)$ is an isomorphism). A minimal model for $(A, d)$ exists and it is unique up to isomorphism. 
Now consider a simply-connected CW-complex $X$. There is an algebra of piecewise polynomial rational differential forms $\left(\Omega_{P L}^{*}(X), d\right)$ defined in [3]. A minimal model of $X$ is a minimal model $\left(\Lambda V_{X}, d\right)$ for $\left(\Omega_{P L}^{*}(X), d\right)$. We have that

$$
\left\{\begin{array}{l}
V^{k}=\left(\pi_{k}(X) \otimes \mathbb{Q}\right)^{*}, \\
H^{k}(\Lambda V, d)=H^{k}(X, \mathbb{Q}) .
\end{array}\right.
$$

\section{Main Theorem}

Let $X$ be a rationally elliptic and simply connected topological space with a Sullivan minimal model $(\Lambda V, d)$, such that $V=\mathbb{Q}\left(a_{1}, \cdots, a_{n}\right)$ with $\left|a_{i}\right|$ are odd integers for all $i, 1 \leq i \leq n$.

Let $\left\{\alpha_{1}, \cdots, \alpha_{r}\right\}$ be a homogeneous basis of $H^{*}\left(\Lambda\left(a_{1}, \cdots, a_{n-1}\right), d\right)$, for all $i, 1 \leq i \leq n$, we put $A_{i}=\Lambda\left(a_{1}, \cdots, a_{i}\right), d a_{i}=P_{i} \in A_{i}$ and for all $k$, $1 \leq k \leq r, \alpha_{k}=\left[\omega_{k}\right]$.

Then we have the following result:

Theorem 3.1. Let $X$ be a rationally elliptic and simply connected topological space with a Sullivan minimal model $(\Lambda V, d)$, where $V=\mathbb{Q}\left(a_{1}, \cdots, a_{n}\right)$ with $\left|a_{i}\right|$ are odd integers. If we have for all $i, 1 \leq i \leq n$, $A_{i}=\Lambda\left(a_{1}, \cdots, a_{i}\right), d a_{i}=P_{i} \in A_{i}$, such that $\left[P_{i}^{2}\right]=0$ in $H^{*}\left(A_{i-1}\right)$, then $\operatorname{dim} H^{*}\left(A_{n}\right) \geqslant n$.

Our goal is to prove that $\operatorname{dim} H^{*}\left(\Lambda\left(a_{1}, \cdots, a_{n}\right), d\right) \geqslant n$ such that $V=\mathbb{Q}\left(a_{1}, \cdots, a_{n}\right)$ with $\left|a_{i}\right|$ odd integers and $\left(d a_{i}\right)^{2}=d Q_{i}$ for all $i$, where $Q_{i} \in \Lambda\left(a_{1}, \cdots, a_{i-1}\right)$. 
We suppose by recurrence that $\operatorname{dim} H^{*}\left(\Lambda\left(a_{1}, \cdots, a_{n-1}\right) \geq n-1\right.$, and let us prove that $\operatorname{dim} H^{*}\left(\Lambda\left(a_{1}, \cdots, a_{n}\right)\right) \geq n$, the case $n=1$ is obvious.

Let $\left\{\alpha_{1}, \cdots, \alpha_{r}\right\}$ be a homogeneous basis of $H^{*}\left(\Lambda\left(a_{1}, \cdots, a_{n-1}\right)\right)$; we put $d a_{n}=P_{n} \in \Lambda\left(a_{1}, \cdots, a_{n-1}\right), \alpha_{k}=\left[\omega_{k}\right], 1 \leq k \leq r$ and $A_{i}=\Lambda\left(a_{1}\right.$, $\left.\cdots, a_{i}\right), 1 \leq i \leq n$.

We have two cases:

Case 1. $\left[P_{n}\right]=0$ in $H^{*}\left(A_{n-1}\right)$ :

Let us write $d a_{n}=P_{n}$. If $\left[P_{n}\right]=0$ and $P_{n}=d Q$, then we can do the following exchange of variables $a_{n}^{\prime}=a_{n}-Q$. In this case $\left(\left(\Lambda\left(a_{1}, \cdots, a_{n}\right)\right.\right.$, $d) \simeq\left(\Lambda\left(a_{1}, \cdots, a_{n-1}\right), d\right) \otimes\left(\Lambda a_{n}^{\prime}, 0\right) \quad$ and $H^{*}\left(\Lambda\left(a_{1}, \cdots, a_{n}\right)\right)=H^{*}\left(\Lambda\left(a_{1}\right.\right.$, $\left.\cdots, a_{n-1}\right) \otimes \Lambda a_{n}^{\prime}$. Therefore $\operatorname{dim} H^{*}\left(\Lambda\left(a_{1}, \cdots, a_{n}\right)\right) \geq 2(n-1)$.

Case 2. $\left[P_{n}\right] \neq 0$ in $H^{*}\left(\Lambda\left(a_{1}, \cdots, a_{n}\right)\right)$ :

Let us write $\Im: A_{n-1} \rightarrow A_{n}$ for the canonic injection and $\Im_{*}: H^{*}\left(A_{n-1}\right)$ $\rightarrow H^{*}\left(A_{n}\right)$ the induced morphism in cohomology.

Proposition 3.2. We have $\operatorname{ker} \Im_{*}=H^{*}\left(A_{n-1}\right) .\left[P_{n}\right]$, which is the ideal generated by $\left[P_{n}\right]$.

Proof. (a) One has $\Im_{*}\left(\left[\omega P_{n}\right]\right)=\left[\omega P_{n}\right]=\left[d\left(\omega a_{n}\right)\right]=0$, hence $H^{*}\left(A_{n-1}\right) \cdot\left[P_{n}\right] \subset \operatorname{ker} \Im_{*}$.

(b) Let $\alpha \in \operatorname{ker} \Im_{*}$, then $\alpha=0$ in $H^{*}\left(A_{n}\right)$ so there exists $\omega$ in $A_{n-1}$ and a polynomial $R$ in $A_{n}$ such that $\omega=d R$ and $[\omega]=\alpha$.

$$
\exists(P, Q) \in A_{n-1}^{2} \text { such that } R=P a_{n}+Q \text { so }\left\{\begin{array}{l}
\alpha=[\omega], \\
d \omega=0, \\
\omega=d\left(P a_{n}+Q\right) .
\end{array}\right.
$$

Then $\omega=(d P) a_{n}+(-1)^{|P|} P P_{n}+d Q$. 
We also have $\omega \in A_{n-1}$ then $\exists\left(P^{\prime}, Q^{\prime}\right) \in A_{n-2}^{2}$ such that $\omega=P^{\prime} a_{n-1}+Q^{\prime}$. Then $\omega=d R=d P a_{n}+(-1)^{|P|} P P_{n}+d Q=P^{\prime} a_{n-1}+Q^{\prime}$ so $\left\{\begin{array}{l}d P=0, \\ \omega=\left((-1)^{|P|} P P_{n}+d Q\right) .\end{array}\right.$

Therefore $\alpha=\left[\omega^{\prime} P_{n}\right]$, where $\left[\omega^{\prime}\right]=\left[(-1)^{|P|} P\right]$ so $\alpha \in H^{*}\left(A_{n-1}\right) \cdot\left[P_{n}\right]$, then $\operatorname{ker} \Im_{*}=H^{*}\left(A_{n-1}\right) \cdot\left[P_{n}\right]$.

Proposition 3.3. One has $\operatorname{dim} H^{*}\left(A_{n}\right) \geq \operatorname{dim} H^{*}\left(A_{n-1}\right)$.

Proof. Let us denote by $B_{1}=\left\{\left[\omega_{i} P_{n}\right]\right.$ such that $1 \leq i \leq p$ and $\left|\omega_{1}\right| \leq \cdots$, $\left.\leq\left|\omega_{p}\right|\right\}$ a basis of $\operatorname{ker} \Im_{*}$, and $B_{2}=\left\{\beta_{j}=\left[\rho_{j}\right] / 1 \leq j \leq q\right\}$ a basis of one complementary $F$ of ker $\Im_{*}$ in $H^{*}\left(A_{n-1}\right)$. Let us prove that $B=\left\{\left[\omega_{i} P_{n}\right.\right.$ $\left.\left.a_{n}-\omega_{i} Q_{n}\right], \beta_{j} / 1 \leq i \leq p, 1 \leq j \leq q\right\}$ is an additively free family in $H^{*}\left(A_{n}\right)$. Indeed, let $\left(\lambda_{1}, \cdots, \lambda_{p}\right) \in \mathbb{Q}^{p}$ and $\left(\mu_{1}, \cdots, \mu_{q}\right) \in \mathbb{Q}^{q}$ be rational coefficients such that $\sum_{i=1}^{p} \lambda_{i}\left[\omega_{i} P_{n} a_{n}-\omega_{i} Q_{n}\right]+\sum_{j=1}^{q} \mu_{j}\left[\rho_{j}\right]=0$. Then $\exists(P, Q) \in A_{n-1}^{2}$ such that $\sum_{i=1}^{p} \lambda_{i} \omega_{i} P_{n} a_{n}-\sum_{i=1}^{p} \lambda_{i} \omega_{i} Q_{n}+\sum_{j=1}^{q} \mu_{j} \rho_{j}=$ $d\left(P a_{n}+Q\right)=(d P) a_{n}+(-1)^{|P|} P P_{n}+d Q$, therefore

$$
\left\{\begin{array}{l}
\sum_{i=1}^{p} \lambda_{i} \omega_{i} P_{n}=d P \\
\sum_{j=1}^{q} \mu_{j} \rho_{j}-\sum_{i=1}^{p} \lambda_{i} \omega_{i} Q_{n}=(-1)^{|P|} P P_{n}+d Q
\end{array}\right.
$$

Hence

$$
\left\{\begin{array}{l}
\sum_{i=1}^{p} \lambda_{i}\left[\omega_{i} P_{n}\right]=0 \Rightarrow \lambda_{i}=0 \\
\sum_{j=1}^{q} \mu_{j} \beta_{j} \in H^{*}\left(A_{n-1}\right) \cdot\left[P_{n}\right] \cap F
\end{array}\right.
$$

Then for all $1 \leq i \leq p, 1 \leq j \leq q,\left(\lambda_{i}, \mu_{j}\right)=(0,0)$, so we can conclude that, $\operatorname{dim} H^{*}\left(A_{n}\right) \geq \operatorname{card}(B)=\operatorname{dim} H^{*}\left(A_{n-1}\right)$. 
Let us denote by $Z\left(A_{n-1}\right):=\operatorname{ker}\left(d: A_{n-1} \rightarrow A_{n-1}\right)$ and $B\left(A_{n-1}\right):=\operatorname{Im}$ $\left(d: A_{n-1} \rightarrow A_{n-1}\right)$, and let us consider the morphism

$$
\begin{gathered}
\phi: H^{*}\left(A_{n-1}\right) \rightarrow H^{*}\left(A_{n-1}\right), \\
{[\omega] \mapsto\left[\omega P_{n}\right]=\left[P_{n} \omega\right] .}
\end{gathered}
$$

Lemma 3.4. One has $\operatorname{Im} \phi \subset \operatorname{ker} \phi$.

Proof. Indeed if $\theta \in Z\left(A_{n-1}\right)$, then $\phi\left(\left[\theta P_{n}\right]\right)=\left[\theta P_{n}^{2}\right]=\left[d\left(\theta Q_{n}\right)\right]=0$.

Lemma 3.5. If $\operatorname{ker} \phi=\operatorname{Im} \phi$, then $\operatorname{dim} H^{*}\left(A_{n}\right) \geq \operatorname{dim} H^{*}\left(A_{n-1}\right)+1$.

Proof. (a) If $\forall 1 \leq k \leq n-1, d a_{k}=0$, then $\operatorname{dim} H^{*}\left(A_{n}\right) \geq \operatorname{dim} H^{*}$ $\left(A_{n-1}\right) \geq 2^{n-1} \geq n$.

(b) If there exists $l \in\{1, \cdots, n-1\}$ such that $d a_{l} \neq 0$, then $d a_{l} \in B$ $\left(A_{n-1}\right) \subset \operatorname{ker} \phi$, hence there exists $\lambda \in \mathbb{Q}^{*}$ such that $d a_{l}=\lambda P_{n}$, since $\left|a_{1}\right| \leq, \cdots, \leq\left|a_{n}\right|$, therefore $\left|d a_{l}\right| \leq\left|P_{n}\right|$.

Let $\beta=\left[a_{l}-\lambda a_{n}\right] \in H^{*}\left(A_{n}\right)$ and let's prove that the family $B \cup\{\beta\}$ is free in $H^{*}\left(A_{n}\right)$.

Indeed, if we suppose by contradiction that $\beta$ can be written as a linear composition of the form

$$
\beta=\left[\sum_{i=1}^{p} \lambda_{i} \omega_{i} P_{n} a_{n}-\sum_{i=1}^{p} \lambda_{i} \omega_{i} Q_{n}+\sum_{j=1}^{q} \mu_{j} \rho_{j}\right], \text { where } \lambda_{i} \text { and } \mu_{j} \text { are }
$$

rational numbers, then $a_{l}-\lambda a_{n}=\sum_{i=1}^{p} \lambda_{i} \omega_{i} P_{n} a_{n}-\sum_{i=1}^{p} \lambda_{i} \omega_{i} Q_{n}+$ $\sum_{j=1}^{q} \mu_{j} \rho_{j}+d Q$, where $Q \in A_{n}$

But this is impossible since the second member does not contain the term $a_{l}$, hence, $\operatorname{dim} H^{*}\left(A_{n}\right) \geq \operatorname{dim} H^{*}\left(A_{n-1}\right)+1$. 
Lemma 3.6. If $\operatorname{ker} \phi \neq \operatorname{Im} \phi$, then $\operatorname{dim} H^{*}\left(A_{n}\right) \geq \operatorname{dim} H^{*}\left(A_{n-1}\right)+1$.

Proof. We know by the Lemma 2.5 that $B$ is an additively free family in $H^{*}\left(A_{n}\right)$, let $[\omega] \in \operatorname{ker} \phi \backslash \operatorname{Im} \phi$.

Then we have $\phi([\omega])=0$.

If there exists $\rho \in A_{n-1}$ such that $\omega P_{n}=d \rho$, let $\alpha=\left[a_{n} \omega-\rho\right] \in H^{*}\left(A_{n}\right)$ and let us prove that $B \cup\{\alpha\}$ is an additively free family in $H^{*}\left(A_{n}\right)$.

Indeed if $\alpha=\left[\sum_{i=1}^{p} \lambda_{i} \omega_{i} P_{n} a_{n}-\sum_{i=1}^{p} \lambda_{i} \omega_{i} Q_{n}+\sum_{j=1}^{q} \mu_{j} \rho_{j}\right]$ with $\lambda_{i}, \mu_{j}$ are rational numbers, then there exist $(P, Q) \in A_{n-1}^{2}$ such that $a_{n} \omega-\rho=$ $a_{n}\left(\sum_{i=1}^{p} \lambda_{i} \omega_{i} P_{n}\right)-\sum_{i=1}^{p} \lambda_{i} \omega_{i} Q_{n}+\sum_{j=1}^{q} \mu_{j} \rho_{j}+d\left(a_{n} P+Q\right)=a_{n}\left(\sum_{i=1}^{p} \lambda_{i}\right.$ $\left.\omega_{i} P_{n}-d P\right)+\sum_{j=1}^{q} \mu_{j} \rho_{j}+P_{n} P+d Q$, we get the following equalities:

$$
\left\{\begin{array}{l}
\omega=\sum_{i=1} \lambda_{i} \omega_{i} P_{n}-d P(\star), \\
\rho=-\left(\sum_{j=1}^{q} \mu_{j} \rho_{j}+P_{n} P+d Q\right)+\sum_{i=1}^{p} \lambda_{i} \omega_{i} Q_{n} .
\end{array}\right.
$$

But $(\star)$ implies that $[\omega] \in \operatorname{Im} \phi,\left([\omega]=\phi\left(\sum_{i=1}^{p} \lambda_{i}\left[\omega_{i}\right]\right)\right)$, which is a contradiction since $[\omega] \in \operatorname{ker} \phi \backslash \operatorname{Im} \phi$.

\section{An Example}

Let $V$ be a graded vector space with a basis $\left\{a_{1}, \cdots, a_{6}\right\}$ such that $a_{1}, a_{2} \in V^{3}, a_{3} \in V^{5}, a_{4}, a_{5} \in V^{7}$, and $a_{6} \in V^{9}$

Now we define a linear map $d$ of degree 1 by: $d a_{1}=d a_{2}=0, d a_{3}=a_{1} a_{2}, d a_{4}=a_{1} a_{3}, d a_{5}=a_{2} a_{3}$, and $d a_{6}=a_{1} a_{5}$ $+a_{2} a_{4}$. 
We have $\left(d a_{6}\right)^{2}=2 a_{1} a_{2} a_{4} a_{5}=d\left(2 a_{3} a_{4} a_{5}\right)$, so $\left[\left(d a_{6}\right)^{2}\right]=0$ in $H^{*}$ $\left(\Lambda\left(a_{1}, \cdots, a_{6}\right), d\right)$.

The family $\left\{[1],\left[a_{1}\right],\left[a_{2}\right],\left[a_{1} a_{4}\right],\left[a_{2} a_{5}\right],\left[a_{1} a_{2} a_{3} a_{4} a_{5} a_{6}\right]\right\}$ is free in $H^{*}\left(\Lambda\left(a_{1}, \cdots, a_{6}\right), d\right)$. Therefore, $\operatorname{dim} H^{*}\left(\Lambda\left(a_{1}, \cdots, a_{6}\right), d\right) \geq 6$.

\section{References}

[1] M. Amann, A note on the Hilali conjecture, Forum Mathematicum 29(2) (2017), 251-257.

DOI: https://doi.org/10.1515/forum-2015-0078

[2] Y. Félix, S. Halperin and J.-C. Thomas, Rational Homotopy Theory, Volume 205 of Graduate Texts in Mathematics, Springer-Verlag, New York, 2001.

DOI: https://doi.org/10.1007/978-1-4613-0105-9

[3] P. A. Griffiths and J. W. Morgan, Rational Homotopy Theory and Differential Forms, Progress in Mathematics, Volume 16, Birkhäuser, 1981.

[4] J. Fernandez de Bobadilla, J. Fresan, V. Munoz and A. Murillo, The Hilali Conjecture for Hyperelliptic Spaces, Mathematics without Boundaries: Surveys in Pure Mathematics, Book Chapter pp. 21-36, 2014.

[5] M. R. Hilali, Actions du tore $T^{n}$ sur les espaces simplement connexes, Thèse à l’Universite Catholique de Louvain, 1990.

[6] M. R. Hilali and M. I. Mamouni, A lower bound of cohomologic dimension for an elliptic space, Topology and its Applications 156(2) (2008), 274-283.

DOI: https://doi.org/10.1016/j.topol.2008.07.011

[7] M. R. Hilali and M. I. Mamouni, A conjectured lower bound for the cohomological dimension of elliptic spaces, Journal of Homotopy and Related Structures 3(1) (2008), 379-384.

[8] M. R. Hilali, M. I. Mamouni and H. Yamoul, On the Hilali conjecture for configuration spaces of closed manifolds, African Diaspora Journal of Mathematics 18(1) (2015), 1-11.

[9] O. Nakamura and T. Yamaguchi, Lower bounds of Betti numbers of elliptic spaces with certain formal dimensions, Kochi Journal of Mathematics 6 (2011), 9-28. 